\title{
Phosphorus and Potassium Fertilizer Application Strategies in Corn-Soybean Rotations
}

\author{
Timothy J. Boring ${ }^{1}$, Kurt D. Thelen ${ }^{2, *}$, James E. Board ${ }^{3}$, Jason L. De Bruin ${ }^{4}$, Chad D. Lee ${ }^{5}$, \\ Seth L. Naeve ${ }^{6}$, William J. Ross ${ }^{7}$, Wade A. Kent ${ }^{8}$ and Landon L. Ries ${ }^{9}$ \\ 1 Michigan Agri-Business Association, East Lansing, MI 48824, USA; boringti@msu.edu \\ 2 Plant, Soil, and Microbial Sciences, Michigan State University, East Lansing, MI 48824, USA \\ 3 Emiritus, Department of Plant, Environmental, and Soil Science, Louisiana State University, Baton Rouge, \\ LA 70803, USA; jboard@agcenter.lsu.edu \\ 4 DuPont Pioneer, Macomb, IL 61455, USA; jason.debruin@pioneer.com \\ 5 Department of Plant and Soil Sciences, University of Kentucky, Lexington, KY 40546, USA; cdlee2@uky.edu \\ 6 Department of Agronomy and Plant Genetics, University of Minnesota, St. Paul, MN 55108, USA; \\ naeve002@umn.edu \\ 7 Department of Crop, Soil, and Environmental Science, University of Arkansas, Little Rock, AR 72204, USA; \\ jross@uaex.edu \\ 8 Becks Hybrids, Urbandale, IA 55032, USA; wkent@beckshybrids.com \\ 9 DuPont Pioneer, Algona, IA 50511, USA; Landon.l.ries@gmail.com \\ * Correspondence: thelenk3@msu.edu; Tel.: +517-355-0271
}

Received: 3 August 2018; Accepted: 17 September 2018; Published: 19 September 2018

check for updates

\begin{abstract}
To determine if current university fertilizer rate and timing recommendations pose a limitation to high-yield corn (Zea mays subsp. mays) and soybean (Glycine max) production, this study compared annual Phosphorous (P) and Potassium (K) fertilizer applications to biennial fertilizer applications, applied at $1 \times$ and $2 \times$ recommended rates in corn-soybean rotations located in Minnesota (MN), Iowa (IA), Michigan (MI), Arkansas (AR), and Louisiana (LA). At locations with either soil test $\mathrm{P}$ or $\mathrm{K}$ in the sub-optimal range, corn grain yield was significantly increased with fertilizer application at five of sixteen site years, while soybean seed yield was significantly increased with fertilizer application at one of sixteen site years. At locations with both soil test $\mathrm{P}$ and $\mathrm{K}$ at optimal or greater levels, corn grain yield was significantly increased at three of thirteen site years and soybean seed yield significantly increased at one of fourteen site years when fertilizer was applied. Site soil test values were generally inversely related to the likelihood of a yield response from fertilizer application, which is consistent with yield response frequencies outlined in state fertilizer recommendations. Soybean yields were similar regardless if fertilizer was applied in the year of crop production or before the preceding corn crop. Based on the results of this work across the US and various yield potentials, it was confirmed that the practice of applying $\mathrm{P}$ and $\mathrm{K}$ fertilizers at recommended rates biennially prior to first year corn production in a corn-soybean rotation does not appear to be a yield limiting factor in modern, high management production systems.
\end{abstract}

Keywords: phosphorous; potassium; corn-soybean rotation; management; production system

\section{Introduction}

Phosphorus $(\mathrm{P})$ and potassium $(\mathrm{K})$ are essential nutrients for corn and soybean, comprising a significant proportion of total fertilizer expenditures, and can be yield limiting in many major crop production areas in the United States. Determining optimum application rates and timings for these fertilizers has been an ongoing research focus for decades and efforts continue to refine recommendations. Perceptions by producers of stagnant crop yield increases, particularly in 
soybeans, have spurred interest in revisiting the role of production inputs in an attempt to determine yield-limiting factors. Previous studies have investigated optimizing factors including planting date, seed treatment, weed management, variety selection, and tillage in a high management scheme, although no studies have explored the role of fertilizer recommendation schemes on a multi-regional scale.

A central tenet of crop fertilizer recommendations is the identification of the soil test critical level, the point below which crop growth and yield will be limited by nutritional deficiency. Specific soil critical test values differ by geographic region, soil characteristics, crop, and environment and emphasize the need for regional specific recommendations. Fertilizer recommendations for soils with nutrient values below the critical level include fertilizer to meet intended crop needs and to elevate soil test levels above the critical point. Terminology differs by state for soils testing above the critical level, with soils classified as either optimum or medium. Soils in this range are expected to have a good probability of not responding in yield increases with the addition of fertilizer. As such, this range is considered the most economical category in which to maintain soil test values.

While the specific numerical delineations of soil test ranges vary by state, the premise of these ranges serving as a guide to quantify the likelihood of economic yield response to fertilizer application is a consistent theme. Fertility experiments conducted in the time following the development of these recommendations have generally validated existing fertility standards. As expected, corn and soybean grain yield increases to applications of $\mathrm{P}$ and $\mathrm{K}$ have generally not been found in soils testing at or above medium ranges [1-5].

In a corn-soybean crop rotation, a biennial fertilizer application of $\mathrm{P}$ and $\mathrm{K}$ preceding soybean has become a common management practice. While fertilizer recommendations have been specifically developed to meet a single year of crop production following a soil sample, multiple year fertilization for corn and soybeans produced on soils testing in an optimum or higher range is a reasonable practice when combining the recommended rates for each crop in one application [6,7]. The lack of documented yield response to fertilizer applications on high testing soils supports fertility management that consists of periodic fertilizer application to maintain optimal soil test levels. On high testing soils, extended intervals between maintenance fertilizer applications may be possible. Dodd and Mallarino (2005) [8] found eight to nine years of non-fertilizer corn and soybean production could be conducted on high $P$ testing soils before yield responses could be seen from fertilizer application. Buah et al. (2000) [2] documented conflicting soybean yield results comparing annual and biennial $\mathrm{P}$ and $\mathrm{K}$ applications at eight site years on Iowa farmer fields, but recorded increased soybean yield from annual $\mathrm{P}$ applications compared to biennial in two of three years on research station plots testing optimum for P. McCallister et al. (1987) [9] measured greater extractable P when applying P fertilizer annually rather than equivalent total applications made every two, three, or six years. Corn grain yields were not responsive to application frequency, but the authors suggested that smaller, more frequent fertilizer applications lead to increase plant available phosphorous.

The objective of this study was to determine if current fertilizer rate and timing recommendations constitute a yield limitation in modern, high yielding corn and soybean rotations across a range of production regions in the United States.

\section{Materials and Methods}

\subsection{Site Description}

Research trials were established at four sites in Minnesota, three sites each in Iowa, Arkansas and Louisiana, and two sites in Michigan in 2009 (Table 1). Experiments were established on sites with a history of corn-soybean rotations in a randomized complete block design, blocked by crop rotation, with four replications. Corn was established in 2009 for rotation to soybeans in 2010. A second rotation cycle was initiated in 2010 adjacent to existing plots; corn was planted in 2010 for rotation to soybean 
in 2011. Corn and soybean production was conducted following local cultural practices for tillage, row spacing, population and variety.

Table 1. Description of soil characteristics at research sites used in this study in the years 2009 to 2011.

\begin{tabular}{|c|c|c|c|c|c|}
\hline Site & Soil Type & Soil Series & $\mathrm{pH}$ & Initial $P^{\mathbf{a}}$ & Initial K \\
\hline & & & & \multicolumn{2}{|c|}{$\mathrm{Mg} \mathrm{kg}^{-1}$} \\
\hline \multicolumn{6}{|l|}{ Minnesota } \\
\hline Delavan & Fostoria loam & Aquic Hapludolls & 6.8 & $9 \mathrm{M}^{\mathrm{b}}$ & $141 \mathrm{H}$ \\
\hline Lamberton & Ves loam & Calcic Hapludolls & 5.5 & $24 \mathrm{VH}$ & $114 \mathrm{M}$ \\
\hline Morris & Tara silt loam & Aquic Hapludolls & 7.9 & $6 \mathrm{~L}$ & $150 \mathrm{H}$ \\
\hline St. Charles & Seaton silt loam & Typic Hapludolls & 6.2 & $14 \mathrm{M}$ & $77 \mathrm{~L}$ \\
\hline \multicolumn{6}{|l|}{ Iowa } \\
\hline Lewis & Marshall silty clay loam & Typic Hapludolls & 6.8 & $9 \mathrm{~L}$ & $160 \mathrm{H}$ \\
\hline Sutherland & Sac silty clay loam & Oxyaquic Hapludolls & 5.9 & $20 \mathrm{O}$ & $198 \mathrm{VH}$ \\
\hline Ames 2009-2010 & Clarion loam & Typic Hapludolls & 7.2 & $9 \mathrm{~L}$ & $128 \mathrm{~L}$ \\
\hline Ames 2010-2011 & Canisteo silty clay loam & Typic Endoaquolls & 7.2 & $11 \mathrm{O}$ & $82 \mathrm{VL}$ \\
\hline \multicolumn{6}{|l|}{ Michigan } \\
\hline Ingham & Capac loam & Aquic Glossudalfs & 6.3 & $37 \mathrm{AO}$ & $149 \mathrm{AO}$ \\
\hline Branch & Matherton loam & Typic Argiaquolls & 6.5 & $25 \mathrm{O}$ & $136 \mathrm{AO}$ \\
\hline \multicolumn{6}{|l|}{ Arkansas } \\
\hline Colt & Calhoun silt loam & Typic Glossaqualfs & 6.3 & $15 \mathrm{~L}$ & $100 \mathrm{M}$ \\
\hline Keiser & Sharkey silty clay & Chromic Epiaquerts & 6.8 & $45 \mathrm{O}$ & $201 \mathrm{AO}$ \\
\hline Rohwer & Henery silt loam & Typic Fragiaqualfs & 7.3 & $26 \mathrm{M}$ & $58 \mathrm{VL}$ \\
\hline \multicolumn{6}{|l|}{ Louisiana } \\
\hline Baton Rouge & Commerce silt loam & Fluvaquentic Endoaquepts & 6.6 & $29 \mathrm{M}$ & $136 \mathrm{~L}$ \\
\hline St. Joseph & Commerce silt loam & Fluvaquentic Endoaquepts & 6.6 & $51 \mathrm{H}$ & $239 \mathrm{H}$ \\
\hline Winnsboro & Gigger silt loam & Typic Fragiudalfs & 6.3 & $31 \mathrm{M}$ & $115 \mathrm{~L}$ \\
\hline
\end{tabular}

${ }^{a}$ Minnesota soil test $\mathrm{P}$ determined by the Olson method at Delavan and Morris and with the Bray-P1 method at Lamberton and St Charles. Iowa and Michigan soil test P determined with the Bray-P1 method. Kentucky, Arkansas and Louisiana soil test $\mathrm{P}$ determined with the Mehlich 3 method. ${ }^{\mathrm{b}}$ Soil test ranges determined by state soil test labs. $\mathrm{AO}$, Above optimum; $\mathrm{H}$, high; L, low; M, medium; O, optimum; VH, very high; VL, very low.

\subsection{Treatment Plans}

Fertility treatments consisted of annual applications of $\mathrm{P}$ and K prior to corn in 2009 and soybeans in 2010 or biennial applications of $\mathrm{P}$ and $\mathrm{K}$ for both corn and soybeans applied preceding the corn crop. Fertilizer rates were determined from state specific fertilizer recommendations according to soil test values and applied at $1 \times$ and $2 \times$ rates in both annual and biennial practices [7,10-12]. Specific fertilizer rates, which varied by site, can be found at Boring, 2013 [13]. Soils testing below optimal or medium were fertilized with specific state recommended rates; crop removal rate combined with additional fertilizer to bring test levels to optimal ranges. Sites testing above the optimal or medium level were fertilized at crop removal rates. These treatments were compared to a control receiving no $\mathrm{P}$ or $\mathrm{K}$ fertilizer. Fertilizer treatments were broadcast and incorporated in the spring with mono-ammonium phosphate (10-52-0) and potassium chloride (0-0-62). Tillage, row spacing, variety, plant population, planting date and harvest date can be referenced at Boring, 2013 [13] in the study done at Michigan State University, East Lansing, Michigan, USA. Nitrogen management was conducted in accordance with local practices.

Fertility rates were determined for each site from the composite of 10-15 cores sampled to a $15 \mathrm{~cm}$ depth. All samples were analyzed in the originating state's university soil testing lab following standard soil testing procedures. Phosphorus analysis was determined in Minnesota with the Olson method [14] at sites with $\mathrm{pH}$ levels of 6.8 and greater, the Bray-P1 method [14] at sites with $\mathrm{pH}$ below 6.8, in Iowa and Michigan with the Bray-P1 method, and Arkansas and Louisiana with the Mehlich 3 method [15]. Potassium analysis was determined by ammonium acetate extraction [16] in Minnesota, Iowa and Michigan and by Mehlich 3 in Arkansas and Louisiana. Soils were categorized as to relative $\mathrm{P}$ and $\mathrm{K}$ test levels according to state fertilizer recommendations. Categorization of soil test ranges varied by state, both the number and names of categories and the degree to which additional soil information is utilized. Soil test classification included texture, cation exchange capacity, subsoil nutrient concentrations and irrigation supplementation. A commonality among all state classifications 
is the delineation between sub-optimal, optimal, and above optimal test levels. Sub-optimal ranges include very low and low in Minnesota, Iowa and Louisiana; below optimal in Michigan; and very low, low, and medium in Arkansas. Optimal ranges include medium in Minnesota and Louisiana and optimal in Iowa, Michigan and Arkansas. Above optimal soil test ranges include high and very high in Minnesota and Iowa, above optimal in Michigan and Arkansas, and high in Louisiana. These three ranges, sub-optimal, optimal, and above optimal, are used for delineation of individual sites in this study (Table 2). Plot width varied between 3.9 and $7.8 \mathrm{~m}$ and ranged from 9.1 and $12.2 \mathrm{~m}$ in length. Soybean seed yields were obtained by machine from plot centers and adjusted to $130 \mathrm{~g} \mathrm{~kg}^{-1} \mathrm{H}_{2} \mathrm{O}$. Corn grain yields were obtained by either hand or machine harvest and adjusted to $150 \mathrm{~g} \mathrm{~kg}^{-1} \mathrm{H}_{2} \mathrm{O}$.

Table 2. Description of site soil test levels used in the study in the years 2009 to 2011.

\begin{tabular}{lcc}
\hline \multicolumn{1}{c}{ Site } & P Range & K Range \\
\hline Ames 2009-2010, Colt, Rohwer & Sub-optimal & Sub-optimal \\
Lewis, Morris & Sub-optimal & Above optimal \\
Ames 2010-2011, Baton Rouge, St. Charles, Winnsboro & Optimal & Sub-optimal \\
Branch, Delavan, Keiser, Sutherland & Optimal & Above optimal \\
Lamberton, St. Joseph & Above optimal & Optimal \\
Ingham & Above optimal & Above optimal \\
\hline
\end{tabular}

\subsection{Statistical Analysis}

Data were analyzed with SAS statistical software (SAS Institute, Inc. 2014. SAS v. 9.3. SAS Institute, Cary, NC, USA). Analysis of variance was performed using PROC MIXED. Coefficient of variance was determined using PROC GLM. Treatment means were considered statistically different at the $p<0.10$ level. The $90 \%$ confidence interval was chosen because it was considered conservative enough to meet the objective of evaluating fertilizer application practices across the broad study area $[17,18]$.

\section{Results}

\subsection{Sites Testing in the Sub-Optimal Range for Soil P and K}

At 10 total site-years testing sub-optimal for both soil $\mathrm{P}$ and $\mathrm{K}$, fertilizer applications significantly increased corn yields in two of five comparisons and soybean yields in one of five. In all cases where a yield response was documented, annual fertilizer applications at the $1 \times$ rate maximized yield of corn. At Ames, corn grain yield was significantly increased in 2009 by all fertilizer treatments (Table 3) and the annual and biennial $1 \times$ rates resulted in significantly greater yield than the $2 \times$ biennial treatment. Corn grain yield in 2010 was significantly increased at Colt by $1 \times$ annual and $2 \times$ biennial fertilizer treatments compared to the control and $1 \times$ biennial treatments. At Colt, the corn yield in 2009 was not influenced by fertilizer treatments. At Rowher, the $2 \times$ biennial fertilizer treatment significantly reduced 2010 corn grain yield compared to the control. Yield for all other fertilizer treatments were similar to the control. No differences in corn grain yield were observed at Rohwer in 2009. Soybean grain yield was significantly increased by annual $2 \times$ and biennial $1 \times$ fertilizer applications compared to the biennial $2 \times$ and control treatments at Ames in 2009. Soybean seed yield was not affected by fertilizer applications in 2010 or 2011 at Colt and Rohwer locations. At Colt following the 2010-2011 rotation, soil test $\mathrm{P}$ and $\mathrm{K}$ were significantly increased with biennial $2 \times$ application compared to all other treatments (Table 3). Following the 2010-2011 rotation at Rowher, all fertilizer treatments significantly increased soil test $\mathrm{K}$ compared to the control. Fertilizer rates at the $2 \times$ rate significantly increased soil test $\mathrm{K}$ compared to $1 \times$ rates. 
Table 3. Corn and soybean seed yield and soil test results affected by fertilizer treatments at sites with sub-optimal $\mathrm{P}$ and sub-optimal $\mathrm{K}$.

\begin{tabular}{|c|c|c|c|c|c|c|c|c|}
\hline \multirow[t]{2}{*}{ Site } & \multirow[t]{2}{*}{ Crop Rotation } & \multirow[t]{2}{*}{ Control } & \multicolumn{2}{|c|}{ Annual } & \multicolumn{2}{|c|}{ Biennial } & \multirow[t]{2}{*}{$P>F$} & \multirow[t]{2}{*}{ CV } \\
\hline & & & $1 \times$ & $2 \times$ & $1 \times$ & $2 \times$ & & \\
\hline & & \multicolumn{7}{|c|}{ Grain yield $\left(\mathrm{Mg} \mathrm{ha}^{-1}\right)$} \\
\hline \multirow[t]{2}{*}{ Ames, IA } & 2009 Corn & $10.39 c^{*}$ & $12.89 \mathrm{a}$ & $12.29 \mathrm{a}, \mathrm{b}$ & $12.91 \mathrm{a}$ & $11.54 \mathrm{~b}$ & 0.0026 & 6.37 \\
\hline & 2010 Soybean & $3.62 \mathrm{~b}$ & $4.00 \mathrm{a}, \mathrm{b}$ & $4.03 \mathrm{a}$ & $4.02 \mathrm{a}$ & $3.80 \mathrm{~b}$ & 0.0132 & 4.11 \\
\hline \multirow[t]{4}{*}{ Colt, AK } & 2009 Corn & 7.32 & 7.98 & 7.51 & 7.53 & 6.90 & 0.9090 & 21.44 \\
\hline & 2010 Soybean & 2.62 & 2.56 & 2.49 & 2.26 & 2.43 & 0.6031 & 13.16 \\
\hline & 2010 Corn & $5.32 \mathrm{~b}$ & $6.29 \mathrm{a}$ & $5.80 \mathrm{a}, \mathrm{b}$ & $5.28 \mathrm{~b}$ & $6.04 \mathrm{a}$ & 0.0856 & 10.51 \\
\hline & 2011 Soybean & 3.80 & 3.83 & 3.78 & 4.10 & 4.03 & 0.2233 & 5.77 \\
\hline \multirow[t]{5}{*}{ Rohwer, AK } & 2009 Corn & 12.73 & 11.79 & 11.87 & 12.27 & 11.81 & 0.7851 & 11.45 \\
\hline & 2010 Soybean & 2.95 & 3.05 & 3.31 & 3.11 & 3.02 & 0.4121 & 8.66 \\
\hline & 2010 Corn & $9.38 \mathrm{a}$ & $9.10 \mathrm{a}$ & $8.82 \mathrm{a}, \mathrm{b}$ & $9.36 \mathrm{a}$ & $8.50 \mathrm{~b}$ & 0.0293 & 4.55 \\
\hline & 2011 Soybean & 2.28 & 2.38 & 2.21 & 2.11 & 2.28 & 0.3412 & 8.10 \\
\hline & & \multicolumn{7}{|c|}{ Soil test results $\left(\mathrm{Mg} \mathrm{ha}^{-1}\right)$} \\
\hline \multirow[t]{2}{*}{ Colt, AK } & 2010-2011 P & $29 \mathrm{~b}$ & $32 \mathrm{~b}$ & $33 \mathrm{~b}$ & $31 \mathrm{~b}$ & $39 \mathrm{a}$ & 0.0255 & 11.48 \\
\hline & $2010-2011 \mathrm{~K}$ & $94 \mathrm{~b}$ & $96 \mathrm{~b}$ & $100 \mathrm{~b}$ & $94 \mathrm{~b}$ & $106 \mathrm{a}$ & 0.0277 & 5.18 \\
\hline \multirow[t]{2}{*}{ Rohwer, AK } & 2010-2011 P & 37 & 42 & 49 & 41 & 48 & 0.1546 & 14.90 \\
\hline & $2010-2011 \mathrm{~K}$ & $75 c$ & $103 \mathrm{~b}$ & $116 \mathrm{a}$ & $94 \mathrm{~b}$ & $117 \mathrm{a}$ & 0.0004 & 8.74 \\
\hline
\end{tabular}

${ }^{*}$ Letters following numbers within a row represent differences between treatments at the $p \leq 0.10$ level.

\subsection{Sites Testing in the Sub-Optimal Range for $P$ and Above Optimal Range for $K$}

There were eight site-years with suboptimal P and optimal soil test K. All fertilizer treatments significantly increased corn grain yield compared to the control at Lewis in 2009 (Table 4). No significant differences in corn grain yield were observed at Lewis in 2010 or at Morris in 2009 or 2010. Soybean seed yield was not affected by fertilizer treatment at either site. No significant differences in soil test P or K levels were noted at either site following 2009-2010 or 2010-2011 crop rotations (Table 4).

Table 4. Corn and soybean grain yield and soil test results affected by fertilizer treatments at sites with sub-optimal $\mathrm{P}$ and above optimal $\mathrm{K}$.

\begin{tabular}{|c|c|c|c|c|c|c|c|c|}
\hline \multirow[t]{2}{*}{ Site } & \multirow[t]{2}{*}{ Crop Rotation } & \multirow[t]{2}{*}{ Control } & \multicolumn{2}{|c|}{ Annual } & \multicolumn{2}{|c|}{ Biennial } & \multirow[t]{2}{*}{$\mathbf{P}>\mathbf{F}$} & \multirow[t]{2}{*}{$\mathrm{CV}$} \\
\hline & & & $1 \times$ & $2 \times$ & $1 \times$ & $2 \times$ & & \\
\hline & & \multicolumn{7}{|c|}{ Grain yield $\left(\mathrm{Mg} \mathrm{ha}^{-1}\right)$} \\
\hline \multirow[t]{4}{*}{ Lewis, IA } & 2009 Corn & $11.88 b^{*}$ & $13.78 \mathrm{a}$ & $13.98 \mathrm{a}$ & $14.06 \mathrm{a}$ & $14.52 \mathrm{a}$ & 0.0014 & 5.02 \\
\hline & 2010 Soybean & 4.07 & 4.19 & 4.03 & 4.27 & 4.18 & 0.4448 & 4.61 \\
\hline & 2010 Corn & 13.30 & 13.19 & 13.52 & 12.98 & 12.91 & 0.7904 & 5.76 \\
\hline & 2011 Soybean & 4.32 & 4.16 & 4.41 & 4.46 & 4.32 & 0.1176 & 3.10 \\
\hline \multirow[t]{5}{*}{ Morris, MN } & 2009 Corn & 9.73 & 10.41 & 10.83 & 10.51 & 11.26 & 0.4766 & 11.38 \\
\hline & 2010 Soybean & 3.01 & 3.16 & 2.98 & 3.14 & 3.06 & 0.7899 & 7.74 \\
\hline & 2010 Corn & 14.77 & 14.91 & 14.97 & 14.81 & 15.14 & 0.7201 & 2.69 \\
\hline & 2011 Soybean & 2.34 & 3.21 & 2.68 & 2.92 & 2.82 & 0.3405 & 20.48 \\
\hline & & \multicolumn{7}{|c|}{ Soil test results $\left(\mathrm{Mg} \mathrm{ha}^{-1}\right)$} \\
\hline \multirow[t]{2}{*}{ Lewis, IA } & 2010-2011 P & 28 & 39 & 40 & 32 & 27 & 0.5467 & 45.58 \\
\hline & 2010-2011 K & 196 & 252 & 213 & 240 & 195 & 0.1098 & 14.47 \\
\hline \multirow[t]{4}{*}{ Morris, $\mathrm{MN}$} & $2009-2010 \mathrm{P}$ & 7 & 9 & 6 & 5 & 4 & 0.4408 & 58.29 \\
\hline & 2009-2010 K & 156 & 157 & 152 & 152 & 144 & 0.9076 & 14.56 \\
\hline & 2010-2011 P & 9 & 11 & 10 & 9 & 9 & 0.8148 & 27.63 \\
\hline & 2010-2011 K & 165 & 170 & 164 & 169 & 166 & 0.8985 & 6.65 \\
\hline
\end{tabular}

${ }^{*}$ Letters following numbers within a row represent differences between treatments at the $p \leq 0.10$ level.

\subsection{Sites Testing in the Optimal Range for P and Sub-Optimal Range for $K$}

There were 14 comparisons with optimal soil test $\mathrm{P}$ and sub-optimal soil test $\mathrm{K}$. A fertilizer treatment significantly increased yields in only one site-year for each corn and soybean. At Baton Rouge in 2009, all fertilizer treatments resulted in significantly higher corn yield compared to the control (Table 5). Yields were significantly greater for biennial $1 \times$ and $2 \times$ treatments compared to the annual treatment. No significant differences in corn grain yield were observed at other sites testing in the optimal range for P and sub-optimal range for K. In 2010 at Baton Rouge, soybean seed yields were significantly lower with annual and biennial $2 \times$ treatments compared to the control. Soybean seed 
yield did not significantly differ between fertilizer treatments at other sites. At Baton Rouge, soil test $P$ levels following the 2010-2011 crop rotation were highest with the biennial $2 \times$ treatment compared to all other treatments (Table 5). Additionally, soil test P levels were significantly greater with annual $1 \times$, annual $2 \times$, and biennial $1 \times$ treatments compared to the control. Soil test $\mathrm{P}$ and $\mathrm{K}$ following the 2009-2010 rotation and soil test $\mathrm{K}$ following the 2010-2011 rotation did not differ by fertilizer treatment at Baton Rouge. At Ames 2010-2011 and St. Charles, no differences in soil test P and K were observed.

Table 5. Corn and soybean grain yield and soil test results affected by fertilizer treatments at sites with optimal P and sub-optimal K.

\begin{tabular}{|c|c|c|c|c|c|c|c|c|}
\hline \multirow[t]{2}{*}{ Site } & \multirow[t]{2}{*}{ Crop Rotation } & \multirow[t]{2}{*}{ Control } & \multicolumn{2}{|c|}{ Annual } & \multicolumn{2}{|c|}{ Biennial } & \multirow[t]{2}{*}{$\mathbf{P}>\mathbf{F}$} & \multirow[t]{2}{*}{ CV } \\
\hline & & & $1 \times$ & $2 \times$ & $1 \times$ & $2 \times$ & & \\
\hline & & \multicolumn{7}{|c|}{ Grain yield $\left(\mathrm{Mg} \mathrm{ha}^{-1}\right)$} \\
\hline \multirow[t]{2}{*}{ Ames, IA } & 2010 Corn & 12.31 & 12.38 & 13.17 & 13.13 & 13.73 & 0.1405 & 6.94 \\
\hline & 2011 Soybean & 3.48 & 3.83 & 3.62 & 3.73 & 3.86 & 0.9278 & 20.26 \\
\hline \multirow[t]{4}{*}{ Baton Rouge, LA } & 2009 Corn & $3.56 c^{*}$ & $4.73 \mathrm{a}, \mathrm{b}$ & $4.45 \mathrm{~b}$ & $5.12 \mathrm{a}$ & $5.20 \mathrm{a}$ & 0.0068 & 11.67 \\
\hline & 2010 Soybean & $4.32 \mathrm{a}, \mathrm{b}$ & $3.94 \mathrm{~b}, \mathrm{c}$ & $3.76 \mathrm{c}$ & $4.36 \mathrm{a}$ & $3.71 \mathrm{c}$ & 0.0752 & 9.68 \\
\hline & 2010 Corn & 6.33 & 8.57 & 8.62 & 7.92 & 8.12 & 0.1693 & 12.46 \\
\hline & 2011 Soybean & 2.74 & 2.58 & 2.88 & 2.68 & 2.58 & 0.7907 & 14.47 \\
\hline \multirow[t]{4}{*}{ St. Charles, MN } & 2009 Corn & 12.10 & 13.29 & 12.52 & 13.02 & 13.11 & 0.1372 & 4.61 \\
\hline & 2010 Soybean & 3.36 & 3.48 & 3.61 & 3.16 & 3.51 & 0.4408 & 10.07 \\
\hline & 2010 Corn & 12.76 & 12.52 & 13.19 & 12.80 & 12.58 & 0.8802 & 7.70 \\
\hline & 2011 Soybean & 3.32 & 3.28 & 3.47 & 3.34 & 3.26 & 0.6177 & 5.78 \\
\hline \multirow[t]{5}{*}{ Winnsboro, LA } & 2009 Corn & 11.23 & 11.26 & 11.44 & 11.48 & 10.86 & 0.9181 & 9.29 \\
\hline & 2010 Soybean & 4.10 & 4.03 & 4.43 & 4.57 & 4.63 & 0.2777 & 10.36 \\
\hline & 2010 Corn & 10.76 & 10.41 & 10.95 & 11.13 & 10.68 & 0.4588 & 5.70 \\
\hline & 2011 Soybean & 2.06 & 1.23 & 2.15 & 1.87 & 1.66 & 0.1494 & 28.56 \\
\hline & & \multicolumn{7}{|c|}{ Soil test results $\left(\mathrm{Mg} \mathrm{ha}^{-1}\right)$} \\
\hline \multirow[t]{2}{*}{ Ames, IA } & 2010-2011 P & 16 & 20 & 27 & 21 & 34 & 0.2086 & 49.28 \\
\hline & $2010-2011 \mathrm{~K}$ & 143 & 58 & 176 & 167 & 199 & 0.1097 & 17.28 \\
\hline \multirow[t]{4}{*}{ Baton Rouge, LA } & $2009-2010 \mathrm{P}$ & 54 & 56 & 53 & 48 & 54 & 0.2606 & 9.62 \\
\hline & $2009-2010 \mathrm{~K}$ & 230 & 244 & 222 & 215 & 228 & 0.1250 & 6.59 \\
\hline & 2010-2011 P & $47 \mathrm{c}$ & $52 \mathrm{~b}$ & $54 \mathrm{~b}$ & $53 \mathrm{~b}$ & $59 \mathrm{a}$ & 0.0033 & 6.39 \\
\hline & $2010-2011 \mathrm{~K}$ & 229 & 230 & 230 & 216 & 234 & 0.6141 & 7.73 \\
\hline \multirow[t]{4}{*}{ St. Charles, MN } & 2009-2010 P & 6 & 6 & 6 & 6 & 7 & 0.9577 & 41.25 \\
\hline & $2009-2010 \mathrm{~K}$ & 72 & 79 & 77 & 73 & 71 & 0.5816 & 10.71 \\
\hline & 2010-2011 P & 5 & 6 & 5 & 5 & 6 & 0.4382 & 29.68 \\
\hline & $2010-2011 \mathrm{~K}$ & 78 & 71 & 70 & 79 & 74 & 0.4112 & 10.17 \\
\hline
\end{tabular}

${ }^{*}$ Letters following numbers within a row represent differences between treatments at the $p \leq 0.10$ level.

\subsection{Sites Testing for the Optimal Range for $P$ and Above Optimal Range in $K$}

There were 15 comparisons with optimal soil test $\mathrm{P}$ and above optimal soil test $\mathrm{K}$. A fertilizer treatment significantly increased corn grain yields in three of seven comparisons and soybean seed yield in one of eight site-years. Corn grain yield was significantly increased by any fertilizer application at Branch 2009, Delavan in 2010 and Sutherland in 2010 (Table 6). All fertilizer applications at Branch in 2009 and Delavan in 2010 resulted in significantly higher corn grain yield compared to the untreated control. At Sutherland in 2010, fertilizer application of any kind significantly increased corn yield above that of the control, but yield was significantly lower for the biennial $1 \times$ treatment compared to other fertilizer treatments. Corn grain yield at Delavan in 2009, Keiser in 2009 and 2010, and Sutherland in 2009 were not influenced by fertilizer application. At Branch following the 2009-2010 rotation, all treatments except the biennial $1 \times$ treatment significantly increased soil test $\mathrm{K}$ compared to the control (Table 6). Soil test $\mathrm{K}$ was significantly increased at Branch for $2 \times$ rate treatments compared to $1 \times$ treatments. Soil test $\mathrm{K}$ following the 2010-2011 rotation and soil test $\mathrm{P}$ following the 2009-2010 and 2010-2011 crop rotations did not differ by fertilizer treatment. At Delavan, soil test P and K following 2009-2010 and 2010-2011 rotations were not affected by fertilizer treatment. No significant differences in soil test $\mathrm{P}$ or K were observed following the 2010-2011 crop rotation at Keiser. Following the 2010-2011 crop rotation at Sutherland, all fertilizer treatments increased soil test P compared to the control. All fertilizer treatments except the biennial $1 \times$ treatment increased soil test $\mathrm{K}$ compared to the control. Soil test $\mathrm{P}$ and $\mathrm{K}$ was significantly greater for $2 \times$ rate treatments compared to $1 \times$ rate treatments. 
Table 6. Corn and soybean grain yield and soil test results affected by fertilizer treatments at sites with optimal $\mathrm{P}$ and above optimal $\mathrm{K}$.

\begin{tabular}{|c|c|c|c|c|c|c|c|c|}
\hline \multirow[t]{2}{*}{ Site } & \multirow[t]{2}{*}{ Crop Rotation } & \multirow[t]{2}{*}{ Control } & \multicolumn{2}{|c|}{ Annual } & \multicolumn{2}{|c|}{ Biennial } & \multirow[t]{2}{*}{$\mathbf{P}>\mathrm{F}$} & \multirow[t]{2}{*}{$\mathrm{CV}$} \\
\hline & & & $1 \times$ & $2 \times$ & $1 \times$ & $2 \times$ & & \\
\hline & & \multicolumn{7}{|c|}{ Grain yield $\left(\mathrm{Mg} \mathrm{ha}^{-1}\right)$} \\
\hline \multirow[t]{3}{*}{ Branch, MI } & 2009 Corn & $5.36 b^{*}$ & $6.86 \mathrm{a}$ & $6.99 \mathrm{a}$ & $7.01 \mathrm{a}$ & $7.21 \mathrm{a}$ & 0.0013 & 7.46 \\
\hline & 2010 Soybean & 2.70 & 2.78 & 2.86 & 2.83 & 2.89 & 0.9775 & 16.26 \\
\hline & 2011 Soybean & 0.94 & 0.86 & 1.06 & 1.04 & 0.95 & 0.6076 & 20.43 \\
\hline \multirow[t]{4}{*}{ Delavan, MN } & 2009 Corn & 12.50 & 13.59 & 13.45 & 14.05 & 13.66 & 0.3965 & 8.11 \\
\hline & 2010 Soybean & 2.72 & 2.73 & 2.97 & 3.00 & 2.94 & 0.5463 & 10.68 \\
\hline & 2010 Corn & $9.79 \mathrm{~b}$ & $12.16 \mathrm{a}$ & $12.82 \mathrm{a}$ & $11.82 \mathrm{a}$ & $12.34 \mathrm{a}$ & 0.0019 & 6.91 \\
\hline & 2011 Soybean & $2.39 \mathrm{~b}$ & $3.04 \mathrm{a}$ & $3.11 \mathrm{a}$ & $3.05 \mathrm{a}$ & $3.12 \mathrm{a}$ & 0.0159 & 9.69 \\
\hline \multirow[t]{4}{*}{ Keiser, AK } & 2009 Corn & 10.04 & 10.72 & 9.98 & 10.13 & 10.16 & 0.6352 & 7.46 \\
\hline & 2010 Soybean & 3.80 & 3.76 & 3.71 & 3.79 & 3.76 & 0.9902 & 7.79 \\
\hline & 2010 Corn & 10.73 & 10.71 & 10.43 & 11.00 & 10.63 & 0.4529 & 3.91 \\
\hline & 2011 Soybean & 4.31 & 4.32 & 4.40 & 4.37 & 4.51 & 0.4181 & 3.53 \\
\hline \multirow[t]{5}{*}{ Sutherland, IA } & 2009 Corn & 13.60 & 14.27 & 14.3 & 14.46 & 14.41 & 0.3242 & 4.51 \\
\hline & 2010 Soybean & 4.65 & 4.60 & 4.66 & 4.82 & 4.63 & 0.5157 & 3.67 \\
\hline & 2010 Corn & $12.61 \mathrm{c}$ & $13.72 \mathrm{a}$ & $13.90 \mathrm{a}$ & $13.20 \mathrm{~b}$ & $13.46 \mathrm{a}$ & 0.0032 & 2.79 \\
\hline & 2011 Soybean & 3.74 & 3.94 & 3.93 & 4.04 & 3.96 & 0.1382 & 3.90 \\
\hline & & \multicolumn{7}{|c|}{ Soil test results $\left(\mathrm{Mg} \mathrm{ha}^{-1}\right)$} \\
\hline \multirow[t]{4}{*}{ Branch, MI } & 2009-2010 P & 69 & 68 & 70 & 69 & 69 & 0.1238 & 0.85 \\
\hline & $2009-2010 \mathrm{~K}$ & $16 \mathrm{c}$ & $28 \mathrm{~b}$ & $42 \mathrm{a}$ & $21 \mathrm{c}$ & $40 \mathrm{a}$ & $<0.0001$ & 18.30 \\
\hline & 2010-2011 P & 42 & 33 & 54 & 36 & 24 & 0.2943 & 48.33 \\
\hline & $2010-2011 \mathrm{~K}$ & 117 & 89 & 126 & 115 & 77 & 0.3497 & 36.15 \\
\hline \multirow[t]{4}{*}{ Delavan, MN } & 2009-2010 P & 7 & 9 & 10 & 9 & 11 & 0.9223 & 67.03 \\
\hline & $2009-2010 \mathrm{~K}$ & 141 & 149 & 148 & 138 & 131 & 0.4673 & 10.68 \\
\hline & $2010-2011 \mathrm{P}$ & 4 & 4 & 8 & 5 & 5 & 0.3789 & 63.46 \\
\hline & $2010-2011 \mathrm{~K}$ & 135 & 136 & 133 & 124 & 130 & 0.7199 & 10.28 \\
\hline \multirow[t]{2}{*}{ Keiser, AK } & 2010-2011 P & 48 & 56 & 54 & 56 & 57 & 0.4226 & 13.19 \\
\hline & 2010-2011 K & 337 & 341 & 338 & 341 & 341 & 0.9981 & 6.77 \\
\hline \multirow[t]{2}{*}{ Sutherland, IA } & $2010-2011 \mathrm{P}$ & $17 \mathrm{~d}$ & $28 \mathrm{~b}, \mathrm{c}$ & $43 a$ & $23 c$ & $34 \mathrm{a}, \mathrm{b}$ & $<0.0001$ & 16.27 \\
\hline & $2010-2011 \mathrm{~K}$ & $188 \mathrm{~d}$ & $218 b, c$ & $241 \mathrm{a}$ & $200 c, d$ & $222 \mathrm{~b}$ & 0.0019 & 7.15 \\
\hline
\end{tabular}

\footnotetext{
* Letters following numbers within a row represent differences between treatments at the $p \leq 0.10$ level.
}

\subsection{Sites Testing in the Optimal Range for Soil P and K}

There were eight comparisons with optimal soil test $P$ and optimal soil test $K$, though fertilizer application had no effect on corn or soybean seed yield (Table 7). At Lamberton, no differences in soil test P or K levels following 2009-2010 and 2010-2011 crop rotations were observed. At St. Joseph following the 2010-2011 crop rotation, the annual $2 \times$ fertilizer application resulted in significantly greater soil test with both $\mathrm{P}$ and $\mathrm{K}$ compared to the control (Table 7).

Table 7. Corn and soybean grain yield and soil test results affected by fertilizer treatments at sites with above optimal P and optimal K.

\begin{tabular}{|c|c|c|c|c|c|c|c|c|}
\hline \multirow[t]{2}{*}{ Site } & \multirow[t]{2}{*}{ Crop Rotation } & \multirow[t]{2}{*}{ Control } & \multicolumn{2}{|c|}{ Annual } & \multicolumn{2}{|c|}{ Biennial } & \multirow[t]{2}{*}{$\mathbf{P}>\mathbf{F}$} & \multirow[t]{2}{*}{$\mathrm{CV}$} \\
\hline & & & $1 \times$ & $2 \times$ & $1 \times$ & $2 \times$ & & \\
\hline & & \multicolumn{7}{|c|}{ Grain yield $\left(\mathrm{Mg} \mathrm{ha}^{-1}\right)$} \\
\hline \multirow[t]{4}{*}{ Lamberton, MN } & 2009 Corn & 8.22 & 8.84 & 10.31 & 10.33 & 9.23 & 0.3951 & 20.14 \\
\hline & 2010 Soybean & 3.45 & 3.64 & 3.73 & 3.63 & 3.48 & 0.3221 & 5.74 \\
\hline & 2010 Corn & 11.82 & 13.00 & 12.81 & 12.33 & 12.99 & 0.2422 & 6.40 \\
\hline & 2011 Soybean & 2.89 & 3.30 & 3.20 & 3.15 & 3.18 & 0.1348 & 6.68 \\
\hline \multirow[t]{5}{*}{ St. Joseph, LA } & 2009 Corn & 11.49 & 11.35 & 11.06 & 11.16 & 11.41 & 0.6394 & 3.67 \\
\hline & 2010 Soybean & 3.58 & 3.89 & 3.84 & 3.80 & 3.79 & 0.6005 & 3.63 \\
\hline & 2010 Corn & 10.29 & 10.19 & 10.71 & 10.53 & 10.39 & 0.4447 & 3.90 \\
\hline & 2011 Soybean & 3.74 & 3.63 & 3.52 & 3.67 & 3.76 & 0.1003 & 3.38 \\
\hline & & \multicolumn{7}{|c|}{ Soil test results $\left(\mathrm{Mg} \mathrm{ha}^{-1}\right)$} \\
\hline \multirow[t]{4}{*}{ Lamberton, $\mathrm{MN}$} & 2009-2010 P & 12 & 12 & 11 & 10 & 12 & 0.9423 & 31.29 \\
\hline & 2009-2010 K & 111 & 107 & 111 & 115 & 121 & 0.7398 & 14.10 \\
\hline & 2010-2011 P & 6 & 8 & 7 & 7 & 6 & 0.2872 & 15.99 \\
\hline & 2010-2011 K & 104 & 111 & 104 & 106 & 110 & 0.3129 & 5.40 \\
\hline \multirow[t]{2}{*}{ St. Joseph, LA } & 2010-2011 P & $43 b$ & $49 b$ & $61 \mathrm{a}$ & $47 \mathrm{~b}$ & $49 b$ & 0.0067 & 10.57 \\
\hline & 2010-2011 K & $172 b$ & $189 b$ & $211 \mathrm{a}$ & $174 b$ & $180 \mathrm{~b}$ & 0.0446 & 9.18 \\
\hline
\end{tabular}

Letters following numbers within a row represent differences between treatments at the $p \leq 0.10$ level. 


\subsection{Sites Testing in the Above Optimal Range for Both P and $K$}

One site had above optimal soil test levels for both $\mathrm{P}$ and $\mathrm{K}$, for a total of four site year comparisons. No differences were measured in corn or soybean seed yield (Table 8). Annual fertilizer application at the $2 \times$ rate significantly increased soil test $P$ following the 2010-2011 rotation compared to all other treatments. No differences were observed in soil test $\mathrm{P}$ following the 2009-2010 crop rotation or soil test $\mathrm{K}$ following the 2009-2010 and 2010-2011 crop rotations.

Table 8. Corn and soybean grain yield and soil test results affected by fertilizer treatments at sites with above optimal $\mathrm{P}$ and above optimal $\mathrm{K}$.

\begin{tabular}{|c|c|c|c|c|c|c|c|c|}
\hline \multirow[t]{2}{*}{ Site } & \multirow[t]{2}{*}{ Crop rotation } & \multirow[t]{2}{*}{ Control } & \multicolumn{2}{|c|}{ Annual } & \multicolumn{2}{|c|}{ Biennial } & \multirow[t]{2}{*}{$\mathbf{P}>\mathbf{F}$} & \multirow[t]{2}{*}{ CV } \\
\hline & & & $1 \times$ & $2 \times$ & $1 \times$ & $2 \times$ & & \\
\hline & & \multicolumn{7}{|c|}{ Grain yield $\left(\mathrm{Mg} \mathrm{ha}^{-1}\right)$} \\
\hline \multirow[t]{5}{*}{ Ingham, MI } & 2009 Corn & 9.58 & 9.6 & 9.68 & 9.61 & 10.23 & 0.9249 & 12.86 \\
\hline & 2010 Soybean & 2.92 & 2.9 & 3.1 & 3.01 & 3.04 & 0.6311 & 7.05 \\
\hline & 2010 Corn & 9.78 & 9.65 & 9.07 & 9.63 & 9.88 & 0.2297 & 5.01 \\
\hline & 2011 Soybean & 2.81 & 2.66 & 2.66 & 2.7 & 2.73 & 0.1842 & 3.47 \\
\hline & & \multicolumn{7}{|c|}{ Soil test results $\left(\mathrm{Mg} \mathrm{ha}^{-1}\right)$} \\
\hline \multirow[t]{4}{*}{ Ingham, MI } & 2009-2010 P & 67 & 67 & 67 & 66 & 67 & 0.7257 & 1.71 \\
\hline & 2009-2010 K & 64 & 69 & 90 & 78 & 89 & 0.1411 & 21.42 \\
\hline & 2010-2011 P & $63 \mathrm{~b}$ & $69 \mathrm{~b}$ & $80 a$ & $59 \mathrm{~b}$ & $66 \mathrm{~b}$ & 0.0415 & 12.54 \\
\hline & 2010-2011 K & 209 & 222 & 238 & 207 & 236 & 0.2243 & 10.17 \\
\hline
\end{tabular}

Letters following numbers within a row represent differences between treatments at the $p \leq 0.10$ level.

\section{Discussion}

\subsection{Fertilizer Recommendations}

Fertilizer recommendations are built upon the principle that soil test values exceeding the critical soil test level are expected to supply adequate nutrients to support optimal economic growth [10]. However, variability in soil test values and crop responses mean yield responses can be observed from fertilizer applications at all soil test levels, although with decreasing frequency as soil test levels increase. Iowa fertilizer recommendations cite the probability of yield responses as $80 \%$ on very low testing sites, $65 \%$ on low testing, $25 \%$ on optimal soils, $5 \%$ for soils in the high range and less than $1 \%$ in the very high range [7]. Bruulsema (2004) [19] noted that, in Ontario, Canada, corn and soybean have been documented to have a $59 \%$ and $49 \%$, respectively, probability of response to fertilizer applications on soils testing in the medium soil test level. Results from this multi-state study generally agree with these response frequencies, both in terms of the greater probability of a corn yield response compared to soybean and the overall likelihood of yield response. Of the 14 site years with soil test $\mathrm{P}$ and $\mathrm{K}$ values in the optimal or higher range, corn yield was significantly increased at three, or $21 \%$ of sites, while soybean yield was significantly increased at one, or $7 \%$ of sites. Mallarino et al. (2011) [20] observed corn grain responses to broadcast $\mathrm{P}$ and $\mathrm{K}$ fertilizer treatments at five sites with soil test $\mathrm{P}$ and $\mathrm{K}$ testing at optimal levels or below. The only site they found to be unresponsive to preplant broadcast fertilizer application tested in the very high range for soil $\mathrm{P}$ and medium for soil $\mathrm{K}$. In our study, sites with soil test levels in the sub-optimal range for at least one nutrient demonstrated a significant response on corn grain yield at five of sixteen site years. Bordoli and Mallarino (1998) [21] observed frequent corn grain yield responses to P fertilizer on low testing sites in Iowa, but not every low testing site responded to fertilizer application. In our study, soybean seed yield was significantly increased with fertilizer application at two of sixteen site years. These responses are less than those predicted in the Iowa Fertilizer recommendations, but follow the trend illustrated by Bruulsema (2004) [19] of an increased frequency of fertilizer response in corn compared to soybean. 


\subsection{Corn Yield after Treatment}

Corn yield increases in response to fertilizer applications, when observed, did not follow trends with rate or timing. At Branch in 2009, Delavan in 2010, and Lewis in 2009, all fertilizer treatments resulted in a similar increase in yield compared to the control. At Baton Rouge in 2009, yield generally increased with increasing fertilizer rate. This was contrasted by high fertilizer rates detrimentally impacting yield at Ames in 2009 and Rowher in 2010. At these locations, the biennial $2 \times$ treatment had significantly lower yields than other fertilizer treatments. At Rowher, yield from the untreated control exceeded the biennial $2 \times$ treatment, indicating a yield reducing effect of fertilizer application rather than a simple lack of response. Fertilizer applications have been previously associated with reductions in crop growth and yield, although generally these responses have been attributed to proximity of fertilizer and seed. Anghinoni and Barber (1980) [22] measured decreasing corn root length with increasing P rate in pots. Heckman and Kamprath (1992) [23] observed decreased early season corn growth and $\mathrm{K}$ accumulation with increased broadcast $\mathrm{K}$ rates, a phenomenon they attributed to high salt concentrations. Numerous site years of phosphorus and potassium rate studies in Arkansas have generally observed a lack of response on high testing soils, response to rate on low testing sites, and agreement with established fertilizer recommendations. Muir and Hedge (2001) [24] measured corn yield reductions when increasing the fertilizer rate from $101 \mathrm{~kg} \mathrm{~K}_{2} \mathrm{O} \mathrm{ha}^{-1}$, the recommended rate, to $202 \mathrm{~kg} \mathrm{~K}_{2} \mathrm{O} \mathrm{ha}^{-1}$, on both a low and a high $\mathrm{K}$ testing site.

\subsection{Soil Test Levels}

Yield responses at several sites failed to follow patterns that could be directly correlated to fertilizer rate. At Colt in 2010 and Sutherland in 2010, yield among the fertilizer treatments was lowest for the $1 \times$ biennial treatment compared to other fertilizer treatments. Fertilizer rates for this treatment were neither the lowest nor highest, precluding high fertilizer rate injury as an explanation. The occurrence of this trend at three sites in different production regions suggests more than a statistical anomaly or protocol errors. Soil test values following soybean did not differ between fertilizer treatments at Branch. At Colt, soil test $\mathrm{P}$ and $\mathrm{K}$ were increased by biennial $2 \times$ applications compared to all other treatments. While fertilizer applications at $2 \times$ rates would be expected to increase soil test values compared to $1 \times$ rates, this effect would be expected to be more pronounced when fertilizer applications were made closer in time to soil sampling. The lack of expected increases in soil test $\mathrm{P}$ and $\mathrm{K}$ levels, particularly the lack of differences in the annual $1 \times$ from the annual $2 \times$, may provide an explanation for differences in yield between these two treatments. At Sutherland in 2010, corn grain yield was maximized with annual $1 \times$, annual $2 \times$, and biennial $2 \times$ fertilizer treatments. Grain yield for the biennial $1 \times$ treatment was greater than the control, but lagged behind these other treatments. Increases in both soil test $\mathrm{P}$ and $\mathrm{K}$ following the 2010-2011 crop rotation generally followed the same pattern of increase as corn grain yield. Initial soil test $K$ levels were in the high range and tested in this same range in 2011. Soil test $P$ values were initially in the low range, but fertilizer treatments with the highest corn yields in 2010 had soil test $P$ levels in the high range when tested following the two-year rotation, medium test values for the $1 \times$ biennial treatment that was associated with lower corn yield in 2010, and low soil test $P$ values for the control. These trends in soil test results seem to explain 2010 corn grain results, but the low yielding biennial $1 \times$ treatment received medium amounts of $\mathrm{P}$ and $\mathrm{K}$ fertilizer in comparison to other treatments. Examples of decreased yield at medium fertilizer rates have been observed by other researchers as well. Muir and Hedge (2002) [25] noted reduced corn grain yield with a $78 \mathrm{~kg} \mathrm{P}_{2} \mathrm{O}_{5} \mathrm{ha}^{-1}$ application compared to $39 \mathrm{~kg} \mathrm{P}_{2} \mathrm{O}_{5} \mathrm{ha}^{-1}, 157 \mathrm{~kg} \mathrm{P}_{2} \mathrm{O}_{5} \mathrm{ha}^{-1}$ and an untreated control at one site. Mozaffari et al. (2012) [1] observed significant corn yield increases with $224 \mathrm{~kg} \mathrm{~K}_{2} \mathrm{O}^{-1}$ compared to the control at a low $\mathrm{K}$ testing site, though a $179 \mathrm{~kg} \mathrm{~K}_{2} \mathrm{O} \mathrm{ha}^{-1}$ rate decreased yield compared to the control. At two medium $\mathrm{K}$ testing sites, yield responses were not statistically significant, but a similar trend of decreased yield with a $179 \mathrm{~kg} \mathrm{~K}_{2} \mathrm{O} \mathrm{ha}^{-1}$ application was apparent. 


\subsection{Soybean Yield}

Soybean yield responses to fertilizer applications were observed at three sites: Ames in 2010 with sub-optimal P and K, Baton Rouge in 2010 with optimal P and sub-optimal K, and Delavan in 2011 with optimal P and above optimal K. All fertilizer applications resulted in similar yields at Delavan, but fertilizer applications at Ames 2009-2010 and Baton Rouge did not always result in yield increases. At Ames 2009-2010, yield was significantly increased by annual $2 \times$ and biennial $1 \times$ treatments compared to the control, but yield from annual $1 \times$ and biennial $2 \times$ treatments were similar to the control. At Baton Rouge, annual $2 \times$ and biennial $2 \times$ fertilizer treatments resulted in significant yield reductions compared to the control. Instances of yield reduction from high rates of broadcast fertilizer are not commonly observed, but have been noted to occur from both $\mathrm{P}$ and $\mathrm{K}$ applications. Ebelhar and Varsa (2000) [26] documented greater soybean yield at $56 \mathrm{~kg} \mathrm{~K} \mathrm{ha}^{-1}$ than at higher K fertilizer rates, suggesting yield sensitivity to salt concentrations. Farmaha et al. (2011) [27] saw decreasing soybean yields with increasing $\mathrm{K}$ application in no-till systems but were unable to identify a definitive cause. They pointed to Ebelhar and Varsa's postulation of salt injury as a likely explanation. In Arkansas, Slaton et al. (2008) [28] observed a slight decrease in soybean yield with $179 \mathrm{~kg} \mathrm{~K}_{2} \mathrm{O} \mathrm{ha}^{-1}$ compared to $90 \mathrm{~kg} \mathrm{~K}_{2} \mathrm{O} \mathrm{ha}^{-1}$. Slaton et al. (2001) [29] observed decreased soybean yield with $134 \mathrm{~kg} \mathrm{P}_{2} \mathrm{O}_{5} \mathrm{ha}^{-1}$ compared to $90 \mathrm{~kg} \mathrm{P}_{2} \mathrm{O}_{5} \mathrm{ha}^{-1}$ applied annually in a soybean-rice rotation. While maximum $\mathrm{P}$ and $\mathrm{K}$ rates at Baton Rouge were less than those applied at other sites, salt injury from high rates of broadcast applied fertilizer appear to have negatively impacted yield.

Soybean yield was influenced by the timing of fertilizer application in the rotation at two of thirty-two site years of soybean. At Baton Rouge in 2010, the biennial $1 \times$ treatment resulted in significantly greater yield than the $1 \times$ annual treatment. Both annual and biennial $2 \times$ fertilizer treatments resulted in significantly lower yield compared to the control. It is possible that the difference in yield between the $1 \times$ annual and $1 \times$ biennial occurred due to a greater time interval between fertilizer application and soybean production in the biennial fertilizer treatment. At Ames in 2010, no differences were observed when comparing annual $1 \times$ and biennial $1 \times$ application, but annual $2 \times$ applications resulted in higher yield compared to biennial $2 \times$.

\subsection{Comparisons of Soil Test Levels}

Soil test levels following each crop rotation did not consistently differ between treatments. When responses in soil test $\mathrm{P}$ or $\mathrm{K}$ were observed, treatments did not tend to have consistent effects. At Branch following the 2009-2010 rotation, Sutherland following the 2010-2011 rotation, and Rowher following the 2010-2011 rotation, soil test $\mathrm{K}$ levels were increased with $2 \times$ application rates compared to $1 \times$ rates. At all three sites, corn yield increases were documented in the first year of the rotation. Soil test $\mathrm{K}$ levels in control treatments at both Branch and Rowher were in the sub-optimal range, suggesting $\mathrm{K}$ levels could pose a yield limiting condition. While $2 \times$ application rates resulted in higher soil test $\mathrm{K}$ levels than $1 \times$ rates, these increased levels were still below the critical level at both sites. Soil test $\mathrm{K}$ levels at Sutherland were initially characterized as very high and continued to test in this range following the 2010-2011 crop rotation.

The effects of fertilizer application timing were not consistent when increases in soil test $\mathrm{P}$ or K were observed. Soil test P levels at Ingham following the 2010-2011 rotation, soil test K levels at Sutherland following the 2010-2011 rotation, and soil test P and K levels at St Joseph following the 2010-2011 rotation were increased by the annual $2 \times$ treatment compared to all other treatments. In contrast, biennial $2 \times$ fertilizer applications resulted in the highest soil test $\mathrm{P}$ and $\mathrm{K}$ at Colt following the 2010-2011 rotation and soil test $P$ following the 2010-2011 rotation at Baton Rouge. Treatment effects on corn grain yield were only observed at the Sutherland and Colt sites. At both sites, the biennial $1 \times$ application resulted in the lowest yield of all fertilizer treatments. Soil test value responses to fertilizer applications were not consistently increased, but, when observed, increases from annual applications tended to be more associated with greater corn grain yield than when soil test values were increased with biennial applications. 


\section{Conclusions}

Results of this study conducted at several locations showed that present fertilizer recommendations meet nutrient needs of corn and soybean. Corn and soybean yield increases in response to fertilizer applications were observed at sites with adequate fertility levels, but these responses were infrequent and in line with expected response frequencies. Corn yields were more responsive to fertilizer timing and rate than soybean. High fertilizer rates in excess of university fertilizer recommendations were observed to, at best, result in no significant increase in corn or soybean seed yield, and, at worst, result in significant yield reductions. These responses serve to reinforce the current widespread practice of biennial fertilizer applications preceding corn, applied at recommended rates, to supply both corn and soybean fertility needs in a high yield environment.

Author Contributions: Conceptualization, methodology and validation were undertaken by J.E.B., C.D.L., S.L.N., W.J.R. and K.D.T. Formal analysis and investigation were undertaken by T.J.B., L.L.R., W.A.K. and J.L.D.B. The original draft was written by T.J.B. and K.D.T. Review and editing were done by C.D.L., S.L.N., W.J.R. Visualization, supervision, project administration and funding acquisition were undertaken by C.D.L., S.L.N., W.J.R. and K.D.T.

Acknowledgments: The authors wish to thank the field personnel at Michigan State University, University of Arkansas, University of Kentucky, University of Minnesota, Iowa State University, and Louisiana State University, for their technical assistance and support in conducting this research. The authors would also like to thank the United Soybean Board for funding this research.

Conflicts of Interest: The authors declare no conflict of interest.

\section{References}

1. Mozaffari, M.; Slaton, N.A.; Hayes, S.; Griffin, B. Corn response to soil applied phosphorus and potassium fertilization in Arkansas. In Sabbe Arkansas Soil Fertility Studies 2011; Wayne, E., Slaton, N.A., Eds.; Research Series; Arkansas Agricultural Experiment Station, University of Arkansas: Fayetteville, AK, USA, 2012; p. 599.

2. Buah, S.S.J.; Polito, T.A.; Killorn, R. No-tillage soybean response to banded and broadcast and direct and residual fertilizer phosphorus and potassium applications. Agron. J. 2000, 92, 657-662. [CrossRef]

3. Webb, J.R.; Mallarino, A.P.; Blackmer, A.M. Effects of residual and annual applied phosphorus on soil test vales and yields of corn and soybean. J. Prod. Agric. 1992, 5, 148-152. [CrossRef]

4. Mallarino, A.P.; Webb, J.R.; Blackmer, A.M. Corn and soybean yields during 11 years of phosphorus and potassium fertilization on a high-testing soil. J. Prod. Agric. 1991, 4, 312-331. [CrossRef]

5. DeMoody, C.J.; Young, J.L.; Kaap, J.D. Comparative response of soybeans and corn to phosphorus and potassium. Agron. J. 1973, 65, 851-855. [CrossRef]

6. Murdock, L.; Schwab, G. 2010-2011 Lime and Nutrient Recommendations; University of Kentucky College of Agriculture: Lexington, KY, USA, 2010.

7. Sawyer, J.E.; Mallarino, A.P.; Killorn, R.; Barnhart, S.K. A General Guide for Crop Nutrient and Limestone Recommendations in Iowa; Publ. PM 1688 (Rev.); Iowa State Univercity Extension and Outreach: Ames, IA, USA, 2011.

8. Dodd, J.R.; Mallarino, A.P. Soil-test phosphorus and crop grain yield response to long-term phosphorus fertilization for corn-soybean rotations. Soil Sci. Soc. Am. J. 2005, 69, 1118-1128. [CrossRef]

9. McCallister, D.L.; Shapiro, C.A.; Raun, W.R.; Anderson, F.N.; Rehm, G.W.; Engelstad, O.P.; Russelle, M.P.; Olson, R.A. Rate of phosphorus and potassium buildup/decline with fertilization for corn and wheat on Nebraska Mollisols. Soil Sci. Soc. Am. J. 1987, 51, 1646-1652. [CrossRef]

10. Vitosh, M.L.; Johnson, J.W.; Mengel, D.B. Tri-State Fertilizer Recommendations for Corn, Soybean, Wheat and Alfalfa; Extension Bulletin E-2267; Michigan State University: East Lansing, MI, USA; Ohio State University: Columbus, OH, USA; Purdue University: West Lafayette, IN, USA, 1995.

11. Espinoza, L.; Slaton, N.; Mozaffari, M. Understanding the Numbers on Your Soil Test Report; Cooperative Extension Service FAS2118; University of Arkansas: Fayetteville, AR, USA, 2012.

12. Kaiser, D.E.; Lamb, J.A.; Eiason, R. Fertilizer Guidelines for Agronomic Crops in Minnesota; University of Minnesota: Minneapolis, MN, USA, 2011. 
13. Boring, T.J. Field Investigations of Foliar Fertilizer Strategies of Soybean Mn deficiency in Michigan and Phosphorus and Potassium Fertilizer Application Strategies in Corn-Soybean Rotations in the United States. Ph.D. Thesis, Michigan State University, East Lansing, MI, USA, 2013.

14. Frank, K.; Beegle, D.; Denning, J. Phosphorus: Recommended Chemical Soil Test Procedures for the North Central Region; North Central Regional Publ. 221 (Rev.); University of Missouri: Columbia, MO, USA, 1998; pp. $21-29$.

15. Mehlich, A. Mehlich 3 soil extractant: A modification of Mehlich 2 extractant. Commun. Soil Sci. Plant Anal. 1984, 15, 1409-1416. [CrossRef]

16. Warncke, D.; Brown, J.R. Potassium and Other Basic Cations: Recommended Chemical Soil Test Procedures for the North Central Region; North Central Regional Publ. 221 (Rev.); University of Missouri: Columbia, MO, USA, 1998; pp. 31-33.

17. Young, F.J.; Hammer, R.D.; Maatta, J.M. Confidence intervals for soil properties based on differing statistical assumptions. Conf. Appl. Stat. Agric. 1992. [CrossRef]

18. Loescher, H.; Ayres, E.; Duffy, P.; Luo, H.; Brunke, M. Spatial variation in soil properties among North American Ecosystems and guidelines for sampling designs. PLoS ONE 2014, 9, e83216. [CrossRef] [PubMed]

19. Bruulsema, T.W. Understanding the science behind fertilizer recommendations. Better Crops Plant Food 2004, 88, 16-19.

20. Mallarino, A.P.; Bergmann, N.; Kaiser, D.E. Corn responses to in-furrow phosphorus and potassium starter fertilizer applications. Agron. J. 2011, 103, 685-694. [CrossRef]

21. Bordoli, J.M.; Mallarino, A.P. Deep and shallow banding of phosphorus and potassium as alternatives to broadcast fertilization for no-till corn. Agron. J. 1998, 90, 27-33. [CrossRef]

22. Anghinoni, I.; Barber, S.A. Phosphorus application rate and distribution in the soil and phosphorus uptake by corn. Soil Sci. Soc. Am. J. 1980, 44, 1041-1044. [CrossRef]

23. Heckman, J.R.; Kamprath, E.J. Potassium accumulation and corn yield related to potassium fertilization rate and placement. Soil Sci. Soc. Am. J. 1992, 56, 141-148. [CrossRef]

24. Muir, J.H.; Hedge, J.A. Corn response to phosphorus and potassium fertilization at different soil test levels. In Arkansas Soil Fertility Studies 2000; Norman, R.J., Chapman, S.L., Eds.; Research Series 480; Arkansas Agricultural Experiment Station, University of Arkansas: Fayetteville, AK, USA, 2001.

25. Muir, J.H.; Hedge, J.A. Corn response to phosphorus and potassium fertilization at different soil test levels. In Sabbe Arkansas Soil Fertility Studies 2001; Wayne, E., Slaton, N.A., Eds.; Research Series 490; Arkansas Agricultural Experiment Station, University of Arkansas: Fayetteville, AK, USA, 2002.

26. Ebelhar, S.A.; Varsa, E.C. Tillage and potassium placement effects on potassium utilization by corn and soybean. Commun. Soil Sci. Plant Anal. 2000, 31, 2367-2377. [CrossRef]

27. Farmaha, B.S.; Ferández, F.G.; Nafziger, E.D. No-till and strip-till soybean production with surface and subsurface phosphorus and potassium fertilization. Agron. J. 2011, 103, 1862-1869. [CrossRef]

28. Slaton, N.A.; DeLong, R.E.; Mozaffari, M.; Shafer, J.; Branson, J. Soybean response to phosphorus and potassium fertilization rate. In Sabbe Arkansas Soil Fertility Studies 2007; Wayne, E., Slaton, N.A., Eds.; Research Series; Arkansas Agricultural Experiment Station, University of Arkansas: Fayetteville, AK, USA, 2008; p. 558.

29. Slaton, N.A.; DeLong, R.E.; Ntamatungiro, S.; Clark, S.D.; Boothe, D.L. Phosphorus fertilizer rate and application time effect on soybean yield. In Arkansas Soil Fertility Studies 2000; Norman, R.J., Chapman, S.L., Eds.; Research Series; Arkansas Agricultural Experiment Station, University of Arkansas: Fayetteville, AK, USA, 2001; p. 480.

(C) 2018 by the authors. Licensee MDPI, Basel, Switzerland. This article is an open access article distributed under the terms and conditions of the Creative Commons Attribution (CC BY) license (http://creativecommons.org/licenses/by/4.0/). 